\title{
DYKE MONITORIN BY THE MEANS OF PERSISTENT SCATTERING INTERFEROMETRY AT THE COAST OF NORTHERN GERMANY
}

\author{
M. Seidel ${ }^{\mathrm{a}}$, P. Marzahn ${ }^{\mathrm{a}}$, R.Ludwig ${ }^{\mathrm{a}}$ \\ a Dept. of Geography, Ludwigs-Maximilians-Universität München, Germany - \\ m.seidel@iggf.geo.uni-muenchen.de
}

Commission VIII, WG VIII/1

KEY WORDS: SAR-Interferometry, Persistent Scattering Interferometry, displacement measurement

\begin{abstract}
:
40 percent of the world's population are presently living in coastal areas or along the main rivers. Taking into account that the vulnerability of these areas is increasing due to sea-level rise and coastal hazards such as storm surges or extreme rainfall events accompanied with floods, the importance of safety structures such as dykes is increasing as well. Hence, a spatial distributed dyke monitoring should be part of a sustainable adaptation strategy.

Due to increasing amount of SAR-data from various satellites with high spatial and temporal coverage, the means of SARinterferometry could be an essential tool to ensure this kind of required monitoring. Given this prospect, Persistent Scattering Interferometry (PSI) will be a very suitable monitoring technique for dyke structures to identify dyke movement with the accuracy of few millimetres. This procedure focuses on pixels that show a stable scattering behaviour in a sequence of multiple SAR-scenes.

In opposition to ground-measurements, the spatial coverage of this technique provides comparable results for different parts of the dyke; furthermore weak segments with particular high movements could be identified in advance. This could prevent future dyke crevasses and help to reduce risks in high-populated areas.

This paper attempts to describe the potential of the PSI technique for a spatial distributed dyke monitoring at the coast in northern Germany. 21 ERS-2 scenes and 16 Envisat ASAR scenes were analysed. Those Scenes cover an area of a sea shore dyke including a flood regulation barrage and results point out the potential for this technique to monitor dyke structures. Even though the observed dyke doesn't show any significant deformation rates, the two datasets show the same signal for the whole dyke.
\end{abstract}

\section{INTRODUCTION}

Due to demographic growth, rising sea levels and the fact that 40 percent of the world's population is living in coastal areas or next to rivers, the importance of flood protection structures like dykes becomes evident. According to the reinsurance company "Munich Re" 28\% (aprox. 28 billion US\$) of the overall losses caused by natural disasters in 2015 are resulting from floods. Assuming that a dyke is not breaking abruptly but is losing its stability in advance, a continuous monitoring could be essential for the disaster risk reduction of dyke crevasses. Earth observation provides an increasingly stronger contribution to disaster prevention. Synthetic Aperture Radar (SAR) Interferometry (InSAR) is a widely used tool to detect ground deformation in the satellite line of sight (LOS). But it is severely affected by errors due to atmospheric or backscattering differences between the relevant acquisitions leading to temporal or spatial decorrelation. Differences in atmospheric conditions lead to a delay in the transmitted wave and change as well as the backscatter contribution in unstable pixels the received phase information, that inaccuracies or errors in the displacement rates occur.

For the purpose of dyke monitoring with an accuracy of few millimeters, it is important to overcome the limitations of InSAR. The Persistent Scatterer Interferometry (PSI) was first introduced by Feretti et al. (2000). This technique uses a sequence of scenes to identify pixels with a stable scattering behavior. These permanent scatterers can be detected by its phase or amplitude stability over longer-time intervals. The ideal persistent scatterer (PS) is for example a corner reflector because of its dominant phase and amplitude contribution to the pixels sum of backscatter information. But also man-made structures like roofs, houses or a staircase on a dyke with dominant scattering structure within the pixel show similar backscattering behavior because of the double-bounce effect. Although PSI provides a more accurate tool to detect ground deformation, it is still affected by other limitations like low density of PS pixels in non-urban areas or the big demand on well matching scenes. In order to find PS pixels in non-urban areas Hooper et al. (2007) developed the Stanford Method for PS (StaMPS) which is capable to detect PS pixels in non-urban areas via spatial correlation of the phase stability in interferograms. With the background of the new Sentinel-1 data that provides a high temporal and spatial coverage, the potential of the PSI technique for detection of dyke deformation with spaceborn C-band SAR-data is shown in this paper. For this purpose C-band ERS-2 and Envisat ASAR time series have been processed with StaMPS to detect the deformation of the Eider dyke and the Eider barrage building at the North Sea coast in Germany. For this study it is expected to find enough PS on the dyke structure to identify its spatially distributed displacement due to subsidence or lifting processes. Furthermore it is expected to see a correlation between the short term periodical displacement of the dyke and the tidal differences between the acquisitions due to different pressure of the waterbody on the dyke's structure. 


\section{STUDY AREA AND SATELLITE DATA}

\subsection{Properties and history of the Eider dyke and its barrage building}

The coast of northern Germany has constantly been affected by storm surges. After the big flood of 1962 that occurred along the north coast of Germany and in the area of Hamburg, the existing dykes were rebuilt with a height of 8 meter above sea level. In addition, a flood barrage was built at the outlet of the river Eider in the North Sea, to prevent floods bringing water into the upper parts of the river Eider and causing severe floods. In 1973 the building of the barrage was finished. Simultaneous new dykes have been built to reclaim new land. They are located in the west of Schleswig-Holstein in Northern Germany at the mouth of the Eider river. The barrage building consists of 5 big flood gates that can be opened and closed depending on the tidal situation. It protects the surrounding area from storm surges and controls the inflow of salt in the Eider water during floods. In the north and south it is connected to the new sea dykes that protect the area from being flooded during flood events or storm surges as well. (Müller, 2013)

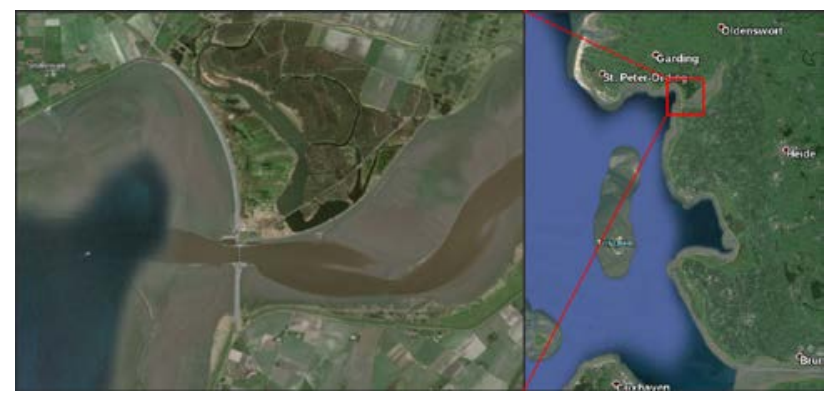

Figure 1: Map of the study area

\subsection{Satellite Dataset}

For the applied analysis, a long time series of ERS-2 and Envisat ASAR data have been chosen. For scene selection the water level at the gauge station in Cuxhaven has been taken into account to assess the correlation between the tidal conditions and the dyke movement. 21 descending ERS-2 scenes and 16 ascending Envisat ASAR scenes were chosen for the analysis.

\begin{tabular}{|c|c|c|c|c|c|}
\hline $\begin{array}{c}\text { Scene } \\
\text { number }\end{array}$ & $\begin{array}{c}\text { Acquisition } \\
\text { date }\end{array}$ & $\mathbf{B}_{\perp}[\mathbf{m}]$ & $\begin{array}{c}\mathbf{B}_{\text {temp }} \\
\text { [days] }\end{array}$ & $\mathbf{f}[\mathbf{H z}]$ & $\begin{array}{c}\text { water } \\
\text { level at } \\
\text { the dike } \\
\text { [cm] }\end{array}$ \\
\hline 1 & $2002-11-16$ & -184.56 & 385 & -10.63 & 565 \\
\hline 2 & $2003-04-05$ & 751.97 & 245 & -99.36 & 378 \\
\hline 3 & $2003-08-23$ & -31.48 & 105 & 21.82 & 621 \\
\hline 4 & $2003-09-27$ & 856.9 & 70 & 44.7 & 348 \\
\hline 5 & $2003-12-06$ & 0 & 0 & 0 & 498 \\
\hline 6 & $2004-02-14$ & -186.52 & -70 & 21.88 & 559 \\
\hline 7 & $2004-04-24$ & -334.58 & -140 & 11.67 & 411 \\
\hline 8 & $2004-05-29$ & -426.5 & -175 & -5.93 & 635 \\
\hline 9 & $2004-07-03$ & 521.6 & -210 & 49.56 & 365 \\
\hline 10 & $2004-08-07$ & 542.51 & -245 & -13.75 & 519 \\
\hline 11 & $2004-10-16$ & -705.79 & -315 & 19.37 & 369 \\
\hline 12 & $2004-12-25$ & 858.58 & -385 & 44.61 & 515 \\
\hline 13 & $2005-01-29$ & -106.76 & -420 & 15.18 & 398 \\
\hline 14 & $2005-07-23$ & 230.09 & -595 & 40.8 & 369 \\
\hline 15 & $2005-11-05$ & -60.95 & -700 & 4.62 & 432 \\
\hline 16 & $2007-04-14$ & -263.23 & -1225 & -34.3 & 585 \\
\hline 1
\end{tabular}

Table 1. List of the Envisat ASAR scenes (ascending)
These scenes fitted the demands of having the same track and perpendicular baselines and Doppler centroid differences being lower than the critical limits (maximal perpendicular baseline = $1100 \mathrm{~m}$, maximal Doppler Centroid difference $=1380 \mathrm{~Hz}$ ), defined by Hooper (2006). The ERS-2 scenes of track 65, acquired between June 1998 and November 2009, contain six scenes representing high tide and 15 scenes representing low tide conditions. To be able to validate the results 16 ASAR scenes with random tidal conditions that had been acquired between November 2002 and April 2007 were also processed. Minimizing the temporal and perpendicular baselines as well as the Doppler centroid from the ERS scenes the $3^{\text {rd }}$ of July 1999 and from the ASAR scenes the $6^{\text {th }}$ of December were chosen as master dates.

\begin{tabular}{|c|c|c|c|c|c|}
\hline $\begin{array}{c}\text { Scene } \\
\text { number }\end{array}$ & $\begin{array}{c}\text { Acquisition } \\
\text { date }\end{array}$ & $\mathbf{B} \perp[\mathrm{m}]$ & $\begin{array}{c}\mathbf{B}_{\text {temp }} \\
{[\text { days] }}\end{array}$ & $\mathbf{f}[\mathbf{H z}]$ & $\begin{array}{c}\text { water } \\
\text { level at } \\
\text { the dike } \\
\text { [cm] }\end{array}$ \\
\hline 1 & $1998-06-13$ & 788.04 & 385 & 16.76 & 346 \\
\hline 2 & $1998-09-26$ & 396.53 & 280 & 0.48 & 348 \\
\hline 3 & $1998-10-31$ & 659.23 & 245 & 65.86 & 687 \\
\hline 4 & $1999-02-13$ & 578.84 & 140 & -10.04 & 615 \\
\hline 5 & $1999-03-20$ & -156.9 & 105 & -52.54 & 337 \\
\hline 6 & $1999-07-03$ & 0 & 0 & 0 & 337 \\
\hline 7 & $1999-10-16$ & -284.3 & -105 & 74.02 & 377 \\
\hline 8 & $2000-04-08$ & 29.18 & -280 & 498.54 & 318 \\
\hline 9 & $2000-07-22$ & -643.28 & -385 & 625.97 & 362 \\
\hline 10 & $2000-09-30$ & -297.73 & -455 & 524.89 & 353 \\
\hline 11 & $2000-12-09$ & -840.15 & -525 & 397.36 & 615 \\
\hline 12 & $2001-01-13$ & 110 & -560 & -30.35 & 315 \\
\hline 13 & $2001-09-15$ & 696.09 & -805 & 250.74 & 659 \\
\hline 14 & $2002-10-05$ & 262.27 & -1190 & -489.52 & 633 \\
\hline 15 & $2004-09-04$ & 388.43 & -1890 & -613.41 & 361 \\
\hline 16 & $2004-11-13$ & -969.39 & -1960 & 312.51 & 626 \\
\hline 17 & $2005-06-11$ & 261.73 & -2170 & -331.42 & 383 \\
\hline 18 & $2006-03-18$ & -262.15 & -2450 & 612.97 & 314 \\
\hline 19 & $2006-07-01$ & -247.38 & -2555 & -62.07 & 358 \\
\hline 20 & $2008-07-05$ & 54.97 & -3290 & -131.88 & 370 \\
\hline 21 & $2009-11-07$ & 194.85 & -3780 & 71.26 & 346 \\
\hline & & & & \\
\hline
\end{tabular}

Table 2. List of ERS-2 scenes (descending)

Precise orbit information from DORIS was used in order to correct the orbital bias. Due to the negligible topography in this region there was no DEM used to correct the topographic phase. In Table 1 and 2 information for the two datasets are summarized, showing the acquisition dates, the perpendicular and temporal baselines, the Doppler Centroid differences with respect to the relevant master images and the water level of the gauge station in Cuxhaven at the moment of acquisition.

\section{THE STAMPS METHOD}

The PSI method was first introduced by Feretti et al. (2000) for InSAR applications. Several authors have developed different approaches since then. An overview can be found in Corsetto et al. (2015). The StaMPS method, introduced by Hooper et al. (2007) was a major improvement detecting PS pixels in nonurban areas using spatial correlation of interferogram phase to find a network of stable pixels. There are mainly four steps to identify PS pixels and estimating their displacements.

Interferograms are generated in StaMPS using a single master image. In comparison to other methods, which have a demand of at least 25 interferograms, to obtain reliable results, for StaMPS 12 interferograms are usually sufficient (Hooper, 
2007). In this step no spectral filtering is applied, in order to maintain the pixel resolution. This increases the chance of the resolution cell being dominated by only one scatterer. Before interferogram formation, the SLC images are co-registered using an amplitude-based algorithm. At the end of this step the position of every pixel in each interferogram is estimated in a geocoded reference frame using orbital parameters.

In order to detect the PS candidates, a combination of amplitude and phase analysis is used, to estimate the probability for each pixel. Initially a first subset of PS pixels is chosen via the amplitude stability in the interferograms. This filtering is done using a threshold criterion on the amplitude dispersion index $\mathrm{D}_{\mathrm{A}}$ (e.g. 0.5), where $\sigma_{\mathrm{A}}$ respresents the amplitudes standard deviation and $\mu_{\mathrm{A}}$ the mean of the amplitude values:

$$
D_{A}=\frac{\sigma_{A}}{\mu_{A}}
$$

These selected PS candidates are then tested for their phase stability. The wrapped phase, $\psi_{\text {int, }, \mathrm{i}, \mathrm{i}}$ of the xth pixel in the ith interferogram consists of different phase contributions and can be written as the sum of 5 terms,

$$
\begin{array}{r}
\psi_{\mathrm{int}, x, i}=W \backslash \backslash\left\{\Phi_{\mathrm{def}, x, i}+\Phi_{a t m, x, i}+\Delta \Phi_{\text {orb }, x, i}+\Delta \Phi_{\theta, x, i}+\right. \\
\left.\Phi_{n, x, i} \backslash \backslash\right\}
\end{array}
$$

where $\Phi_{\text {def, }, \mathrm{i}}$ is the phase change due to the deformation of the pixels surface in the satellite line-of-sight (LOS), $\Delta \Phi_{\theta, \mathrm{x}, \mathrm{i}}$ is the residual phase due to look angle error, $\Phi_{\mathrm{atm}, \mathrm{x}, \mathrm{i}}$ is the phase due to the different atmospheric conditions, $\Delta \Phi_{\mathrm{orb}, \mathrm{x}, \mathrm{i}}$ is the residual phase due to orbit inaccuracies, $\Phi_{\mathrm{n}, \mathrm{x}, \mathrm{i}}$ is a noise term due to the variability in scattering, thermal noise, co-registration inaccuracy and uncertainty in the position of the phase centre and $\mathrm{W}$ is the wrapping operator. Since the four first terms are correlated spatially, they can be estimated using an iterative approach, finding the pixels with small $\Phi_{n, x, i}$. Therefore a measure of the variation of the residual phase for a pixel is defined as

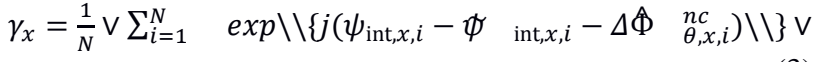

where $N$ is the number of interferograms, $j$ is $\sqrt{-1}$ and $\Delta \hat{\Phi} \quad{ }_{\theta, x, i}^{n c}$ represents the change in the spatially uncorrelated part of the look angle error. For pixel $x$, the probability to be a PS is

$$
P(x \in P S)=1-\frac{(1-\alpha) p_{R}\left(\gamma_{x}\right)}{p\left(\gamma_{x}\right)}
$$

where $0 \leq \alpha \leq 1, p_{R}\left(\gamma_{x}\right)$ is the weighted sum of probability density for PS pixels and $p_{R}\left(\gamma_{x}\right)$ is the probability density for non-PS pixels.

After the PS selection, the wrapped interferogram phase has to be unwrapped. Therefore a three-dimensional approach is used, the third dimension being that of time. First, the temporal phase differences for each PS is calculated and then unwrapped spatially from a reference PS using an iterative least square method. Integrated in time results in an unwrapped phase time series for each PS, with respect to the reference PS. (Hooper, 2006)

\section{RESULTS}

In this section the parameters being used in StaMPS and the results are presented. For both datasets, the ERS-2 and the ASAR data, the same processing parameters have been used as shown in Table 3. The unwrapped interferograms shown in Figure 2 and 3 point out the stable backscattering behaviour of the dyke structure being a concrete man-made structure.

\begin{tabular}{|c|c|}
\hline Parameter & Value \\
\hline Amplitude dispersion threshold & 0.4 \\
\hline Number of patches in range & 3 \\
\hline Number of patches in azimuth & 2 \\
\hline Overlapping pixels between patches (range) & 50 \\
\hline Overlapping pixels between patches (azimuth) & 200 \\
\hline
\end{tabular}

Table 3. Parameters being used for StaMPS processing

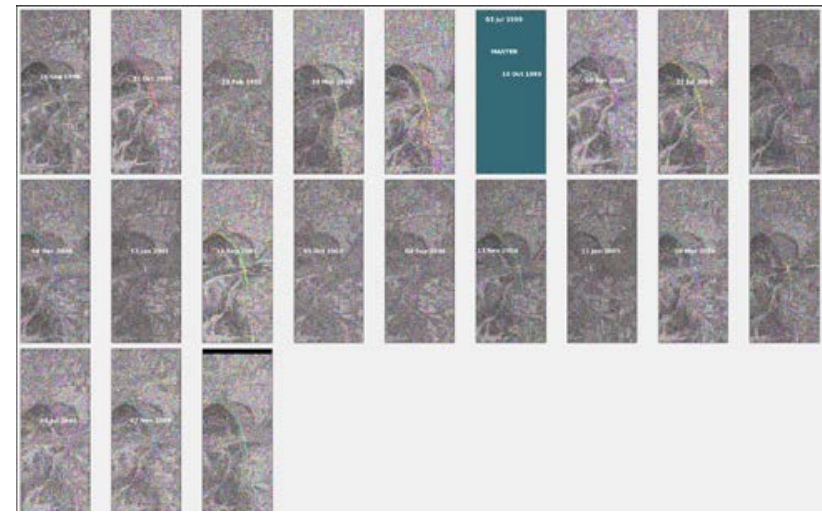

Figure 2. ERS-2 interferograms formed by using a single master scene $(07 / 03 / 1999)$
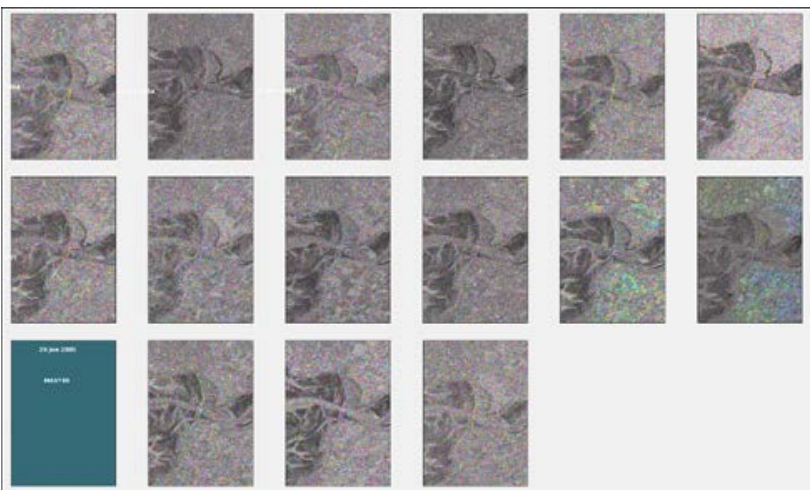

Figure 2. ASAR interferograms formed by using a single master scene $(01 / 29 / 2005)$

The PS displacement plots shown in Figure 4 and 5 indicate the mean annual LOS displacement rates in millimetre per year, while negative values represent movements away from the satellite and positive towards the satellite. In the results obtained from the ASAR scenes 440 PS on the Eider dyke and its barrage building are detected with LOS displacements ranging from -1.2 to $4.1 \mathrm{~mm} / \mathrm{yr}$ and a mean value of $1.5 \mathrm{~mm} / \mathrm{yr}$. In the results obtained from the ERS scenes 254 PS could be detected on the same area, with LOS displacements ranging from -4.3 to -0.6 $\mathrm{mm} / \mathrm{yr}$, with a mean value of $-2.0 \mathrm{~mm} / \mathrm{yr}$. The difference in the number of detected points can be caused by the different look directions resulting in completely different backscatter behaviour. Both sensors were in right-looking mode regarding to the azimuth direction, while the ASAR scenes were acquired in ascending orbit and the ERS-2 scenes in descending orbit. Considering this fact, the different LOS displacement rates are not contradictory, but even support each other's measurement. While normally a negative value in LOS displacement indicates 
subsidence processes and positive values indicate lifting processes, here a vertical deformation can be excluded. Hence the contrary LOS deformation rates can be interpreted as horizontal displacement towards the sea (to the west). Since the whole area shows the same rates, the deformation is most likely caused by tectonical movement or post-glacial compensation.

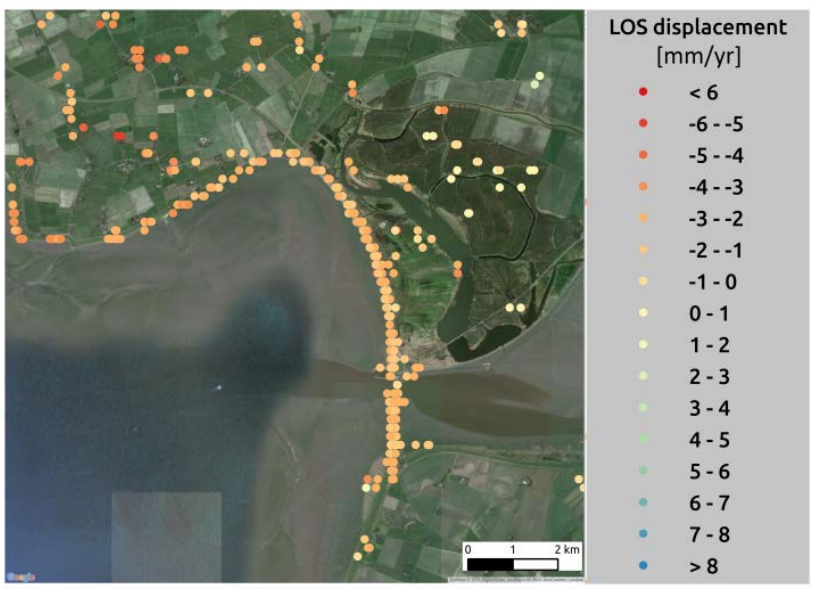

Figure 4. Mean annual LOS displacement rates for the PS at the Eider dyke derived from 21 descending ERS-2 scenes

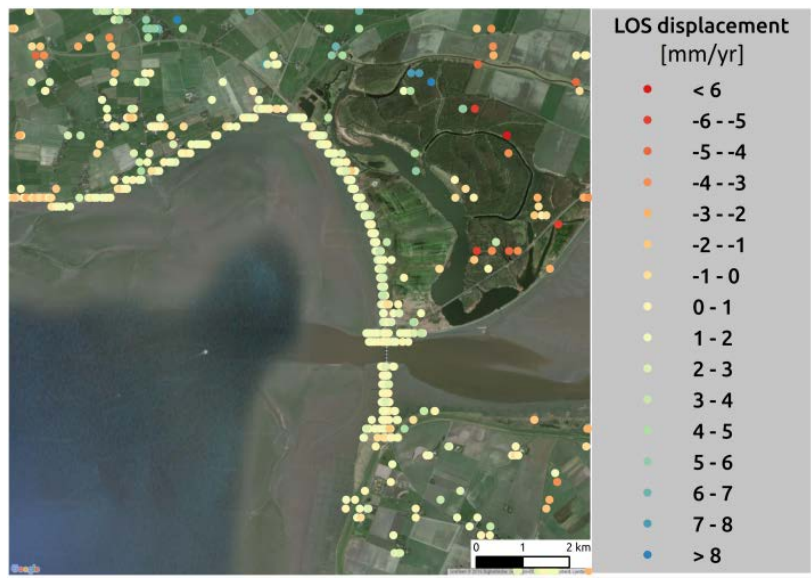

Figure 5. Mean annual LOS displacement rates for the PS at the Eider dyke derived from 16 ascending ASAR scenes

Furthermore the one-dimensional LOS displacement time series can be plotted for each PS. While the mean annual LOS displacement rates from above are the result from a linear regression for each PS which helps to remove errors from single scenes that are eventually not correctly calculated, the time series plots can be analysed for short-term deformation e.g. due to tidal influences. In Figure 6 and 7 an example PS on the dyke is displayed for each dataset. The coloured line represents the surrounding PS in the range of 100 meter. Additionally the letters next to the points show the tidal conditions for the acquisition time $(\mathrm{L}=$ low tide $\leq 400 \mathrm{~cm}, \mathrm{H}=$ high tide $\geq 600 \mathrm{~cm}$, $\mathrm{M}=$ mean tide $400 \mathrm{~cm}<\mathrm{M}<600 \mathrm{~cm}$ ). It was expected to see a small deformation rate with a lifting process or horizontal displacements towards the sea (West) during low tide conditions and vice versa with a subsidence rate or horizontal deformation towards the land (East) during high tide conditions. In the ASAR dataset this should result in a positive LOS displacement during low tide conditions and in a negative LOS displacement during high tide conditions, because the two combined displacement vectors are both facing in the same direction in LOS. For the ERS-2 dataset it should be more difficult to detect deformation caused by tidal differences as the LOS direction vectors are contrary, so that a deformation towards the sea (west) with a vertical uplift could neutralize each other. For the ERS-2 dataset 4 of 6 high tide scenes show positive displacement rates in comparison to the linear regression, while 8 of 14 low tide scenes show negative displacement rates. For the ASAR dataset, where a stronger difference between the tidal conditions was expected, no clear signal could be detected. But considering that this dataset had only 2 high tide and 6 low tide scenes, the validity of this result to show tidal caused displacement differences is low anyway. Interpreting the results from the ERS-2 dataset and assuming that these differences are not caused by atmospheric effects, the vertical process (lifting / subsidence) can be negated, while a tendency for horizontal deformation can be seen. But still the results are not reliable enough to be able to make a clear statement about the above mentioned processes.

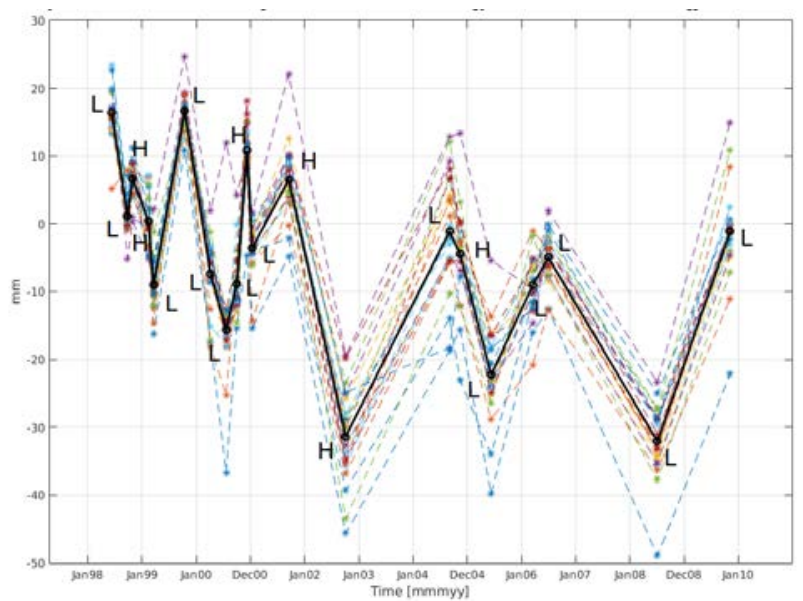

Figure 6. One-dimensional LOS displacement plot for a PS on the Eider dyke, obtained from 21 ERS-2 scenes

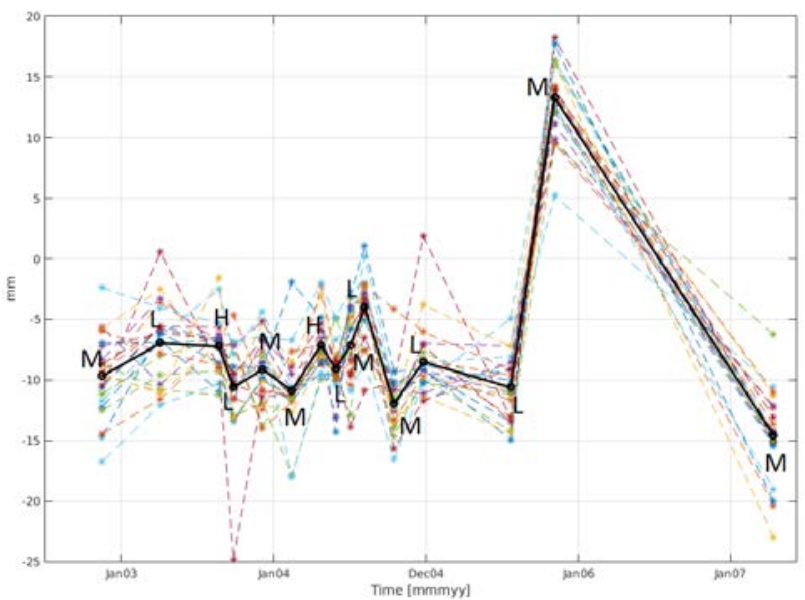

Figure 7. One-dimensional LOS displacement plot for a PS on the Eider dyke, obtained from 16 ASAR scenes 


\section{CONCLUSION}

The StaMPS PSI approach could be shown as a suitable technique to monitor man-made dyke structures. Even though the observed dyke didn't show significant displacement rates, this approach could be an essential tool for flood disaster prevention due to its accuracy. With the help of the time series datasets a long-term deformation can be detected quite well with an accuracy of only few millimetres. Assuming the fact, that a dyke is moving in the area around an imminent crevasse, it should be possible to detect these areas and to prevent such events. This could help to reduce future risks for thousands of people and secure their livelihoods and lives. For the short term deformation caused by tidal differences, only the ERS-2 dataset points out a tendency for horizontal deformations. But to show a direct correlation, the datasets contain not enough scenes. Furthermore the signal is too week and unwrapping errors or errors in the displacement rates in single scenes cannot be excluded, that a clear connection of the deformation and the tidal conditions could not be satisfactorily shown. For the future it is planned to analyse other dykes with bigger datasets, to support the results of this study and to provide also reliable results for the tide induced deformation. From a methodical perspective it is planned to apply the StaMPS Small Baselines Subset (SBAS) approach and its combination with the PSI approach to improve the one dimensional time series analysis. Furthermore the PS selection step could be adapted to dyke structures to be able to also detect spatially distributed deformations on dykes covered with vegetation (grass).

\section{REFERENCES}

Crosetto, M., Monserrat, O., Cuevas-González, M., Devanthéry, N., \& Crippa, B. (2015). Persistent Scatterer Interferometry: A review. ISPRS Journal of Photogrammetry and Remote Sensing.

Ferretti, A., Prati, C., and Rocca F., 2000. Nonlinear subsidence rate estimation using Permanent Scatterers in differential SAR, IEEE Trans. GARS Vol. 38, pp. 2202 -2212, September 2000.

Hooper, A., Zebker, H., Segall, P., \& Kampes, B. (2004). A new method for measuring deformation on volcanoes and other natural terrains using InSAR persistent scatterers. Geophysical research letters, 31(23).

Hooper, A (2006). Persistent scatterer radar interferometry for crustal deformation studies and modeling of volcanic deformation. Dissertation at the department of Geophysics of the Stanford University.

Hooper, A., Segall, P., \& Zebker, H. (2007). Persistent scatterer interferometric synthetic aperture radar for crustal deformation analysis, with application to Volcán Alcedo, Galápagos. Journal of Geophysical Research: Solid Earth, 112(B7).

Hooper, A. (2008). A multi-temporal InSAR method incorporating both persistent scatterer and small baseline approaches, Geophys. Res. Lett., 35, L16302, doi:10.1029/2008GL034654.

Müller, P. (2013). Das Eider-Sperrwerk. Tönning, Germany, Wasser- und Schiffahrtsamt Tönning. http://www.wsv.de/wsatoe/bauwerke/eider_sperrwerk/ (05.04.2016)

Perski, Z., Hanssen, R., Wojcik, A., \& Wojciechowski, T. (2009). InSAR analyses of terrain deformation near the
Wieliczka Salt Mine, Poland. Engineering Geology, 106(1), 5867. 